\title{
Editorial
}

\section{Biomarkers for ovarian cancer: New technologies and targets to address persistently unmet needs}

\author{
Robert C. Bast, Jr.* \\ Department of Experimental Therapeutics, University of Texas M.D. Anderson Cancer Center, Houston, TX, USA
}

Despite significant progress over the last three decades, ovarian cancer remains a formidable adversary. Many articles concerning biomarkers or therapy for this disease begin by pointing to the annual incidence, prevalence and low rate of cure. The fraction of women surviving 5 years has increased from $37 \%$ in 1974-1976 to 46\% in 1999-2005 $(P<0.05)$ [1], but the overall cure rate - the plateau on the survival curvehas not changed despite more widespread utilization of cytoreductive surgery and combination chemotherapy with platinum based compounds and taxanes. The fraction of long term survivors depends not only upon the stage of the disease, but also on the grade of cancer and the ability to remove all, or nearly all, visible tumor nodules at the time of primary surgery. Failure to improve the rate of cure has been attributed to the persistence of dormant drug resistant cancer stem cells and to late diagnosis. Despite substantial research over the last 3 decades, an effective strategy for early detection of ovarian cancer remains an unmet need.

The fact that survival correlates with stage, i.e., the presence and extent of metastasis, has provided a rationale for screening and early detection. When epithelial cancer is diagnosed while still limited to the ovaries (stage I), five year survival rates approach or exceed $90 \%$. Spread to other pelvic organs (stage II) is associated with disease control in up to $70 \%$ of cases. When disease has metastasized to the abdomen (stage III) or

* Address for correspondence: Dr. Bast at Unit 1439, U.T. M.D. Anderson Cancer Center, 1400 Pressler Street, Houston, TX 77030, USA. Tel.: +1 713792 7743; Fax: +1 713792 7864; E-mail: rbast@ ndanderson.org. outside the abdominal cavity (stage IV) the five year survival rate falls to less than $30 \%$. This argument that detection at an earlier stage would improve survival depends critically upon ovarian cancer being a single homogeneous disease that arises in the ovary and that can be detected at different stages of progression and metastasis. Increasingly, it has become apparent that "ovarian cancer" can arise from multiple sites including the Fallopian Tube as well as deposits of endometriosis and is remarkably heterogeneous at the cellular and molecular level.

Pathologists have long recognized different histotypes of ovarian cancer that resemble normal epithelial cells which line the fallopian tube (serous), endometrium (endometrioid), endocervix (mucinous) or which form glycogen-rich rests in the vagina (clear cell). Among the epithelial histotypes, serous cancers account for $>70 \%$. Ovarian cancers also differ in grade with more or less cytoplasmic differentiation and nuclear atypia. The fraction of proliferating cells in different ovarian cancers ranges from less than $5 \%$ to greater than $90 \%$. The prevalence of biomarkers can differ in ovarian cancers of different histotypes, grades and proliferative indices. Once ovarian malignancy is diagnosed, it has become apparent that different targeted therapies will be required for low grade and high grade cancers.

Different molecular abnormalities have been correlated with tumor grade and histotype, prompting a new classification of serous ovarian cancers into two types [2]. Type I cancers exhibit a low histologic grade, low proliferative rate and relative resistance to platinum based therapy. At a molecular level, a significant 
fraction of type I ovarian cancers have activating mutations of Ras and Raf oncogenes, but rarely, if ever, have inactivating mutations of the p53 tumor suppressor. By contrast, type II cancers have high histologic grade, higher proliferative index, and greater sensitivity to chemotherapy. Recent completion of the Cancer Genome Atlas sequence analysis of 316 type II serous cancers indicated that $96 \%$ had inactivating mutations of $\mathrm{p} 53$, but activating mutations of potential oncogenes were uncommon [3]. When abnormalities in gene copy number were analyzed, type II cancers had 116 areas of chromosomal amplification and deletion, reflecting substantial genomic instability, whereas type I cancers have more stable genomes. The $10-15 \%$ of ovarian cancers associated with germ line mutations of BRCA1/2 are generally found among type II tumors. At least $30 \%$ of type II cancers exhibit BRCA dysfunction or BRCAness that could predict response to PARP inhibitors [4]. Among all serous cancers, up to $90 \%$ are type II and as few as $10 \%$ type I. Endometrioid, mucinous and clear cell cancers are sometimes included among type I lesions, but there is a growing consensus that each histotype will require a different strategy for personalized treatment.

Effective screening strategies must take into account the heterogeneity of ovarian cancer. The $30 \%$ of ovarian cancers currently detected in Stage I-II include a disproportionate fraction of low grade type I serous tumors, as well as the less common histotypes. Conversely, the $70 \%$ of ovarian cancers diagnosed in Stage III-IV are more frequently type II high grade serous tumors. Consequently, the more favorable prognosis of early stage cancers could relate to detection of less aggressive disease with a more indolent course, based upon underlying genotypic and phenotypic differences. The challenge for screening is to detect high grade type II serous cancers capable of metastasizing when they are still limited to the ovary or the pelvis.

Given currently available therapy, some authors have argued that the heterogeneity of ovarian cancer will seriously limit the impact of any screening strategy. Type I cancers can, however, be lethal even when they are diagnosed in early stage, can metastasize, and do not account for all stage I disease. Evidence from screening trials conducted in the United Kingdom and in the United States, where a greater fraction of early stage disease has been detected using rising CA125 and transvaginal sonography, suggests that type II high grade serous cancers can be detected while still in early stage [5, 6]. While CA125 can detect a fraction of cancers of each histotype including mucinous and clear cell can- cers, the biomarker is most frequently elevated in blood from patients with serous and endometrioid histotypes. Overall, approximately, $20 \%$ of ovarian cancers fail to express CA125 [7]. If serum biomarkers are to be used as an initial step in screening, panels of ligands will be required to encompass the heterogeneity of all ovarian histotypes, as well as to increase sensitivity for early stage type II serous cancers. One corollary of these assumptions is that panels of biomarkers must be chosen and validated with sera from both early and late stage disease.

In this issue of Cancer Biomarkers, Rodland and Maihle provide an insightful review of the technologies that have been used to discover ovarian cancer biomarkers, as well as a list of the most promising candidates for a multi-marker panel, including CA125, HE4 and mesothelin [8]. High density antibody arrays provide a novel technology to discover other protein biomarkers in serum or plasma as discussed by Ramirez and Lampe [9]. Abbott details alterations in the structure of ovarian cancer associated glycans that could be exploited not only for early diagnosis, but also for therapy [10]. Balch and Nephew review epigenetic changes in ovarian cancer that regulate gene expression, including DNA methylation, modifications to histone proteins, and microRNA post-transcriptional control of protein translation [11]. Alterations in DNA methylation may provide biomarkers for early detection and drug resistance, as well as targets for epigenetic therapy. Histone modification is likely to be particularly important in regulating tumor initiating stem cells. Circulating microRNAs can also serve as diagnostic biomarkers and their survival in blood is likely to depend on packaging in immunosuppressive tumor derived exosomes described by Roberson et al. [12] In considering these many technologies and potential biomarkers for early detection, one obvious challenge is to identify criteria that could prioritize promising biomarkers, as well as to provide banks of sera or other body fluids that could be used to identify and to validate complementary panels.

As noted by Nolen and Lokshin [13], given the prevalence of ovarian cancer in the postmenopausal population (1 in 2500), an effective screening strategy requires not only a high sensitivity $(>75 \%)$, but also a very high specificity $(>99.6 \%)$ to achieve a positive predictive value (PPV) of $10 \%$, i.e. 10 operations for each case of ovarian cancer detected. To achieve sufficiently high specificity, a rising CA125 has been used to trigger transvaginal sonography (TVS) in a small fraction of women screened. The UKCTOCS trial in the United Kingdom utilized this strategy to achieve a 
PPV of $35.1 \%$ in postmenopausal women, with only 3 operations for each case of ovarian cancer detected [5]. A similar PPV of $37.5 \%$ has been obtained in a United States ovarian cancer screening trial sponsored by the Ovarian SPORE at M.D. Anderson Cancer Center using the same risk of ovarian cancer algorithm (ROCA) to detect a rising CA125 and to refer women for TVS [6].

While most strategies have focused on improving specificity to avoid false positive with unnecessary imaging or operations, sensitivity for preclinical asymptomatic disease is also an issue. In the prevalence phase of the UKTOCS trial, a stage shift was observed with $48 \%$ of screen detected cancers in stage I or II, including a number of high grade cancers [5]. The M.D. Anderson Ovarian SPORE study has screened more than 3,000 women over 9 years and performed 9 operations to detect 5 ovarian cancers -3 high grade invasive and 2 borderline - all in stage I or II [6]. As discussed by Nolen and Lokshin, however, Brown and Palmer have modeled the behavior of ovarian cancers from women with mutations in BRCA [14]. In their model, tumor size is correlated with metastatic potential, predicting that effective screens would have to detect cancers of 0.4 to $1.3 \mathrm{~cm}$ diameter. If this prediction can be confirmed in sporadic disease, more sensitive serum biomarkers and imaging techniques would have to be developed.

The sensitivity of tumor derived serum markers depends upon their expression and shedding, as well as their half-life in blood. For biomarkers that are expressed by normal as well as cancer tissues, analyte levels in the presence of cancer must exceed normal levels found in the circulation of healthy individuals. Small volumes of cancer may not shed sufficient amounts of protein, glycan, RNA or DNA to elevate serum biomarker levels. Proteins of $<50 \mathrm{kD}$ are likely to be filtered at the renal glomerulus and might be found in the urine. Biomarkers have been detected in the urine of ovarian cancer patients, including mesothelin [15], Bcl-2 [16] and fragments of CA125 and HE4 [17,18]. In the case of mesothelin, urinary levels correlate with serum levels, but exhibit greater sensitivity. One challenge in the development of urine biomarkers for early detection of ovarian cancer is lack of banked samples from pre-diagnostic cases comparable to the serum banks maintained by the PLCO and the UKCTOCS trials.

Autoantibodies evoked by tumor associated proteins could also provide more sensitive biomarkers for early detection. Chatterjee and Tainsky point out that au- toantibodies might be formed in response to very small volumes of disease and would exhibit a long serum half life relative to conventional protein biomarkers [19]. In studies to date, autoantibodies reactive with MUC1, MUC16 (CA125), IL-8, mesothelin, testis associated antigens and a large number of other tumor associated proteins have been detected in sera from ovarian cancer patients. As virtually all type II high grade serous carcinomas have undergone mutation of p53, autoantibodies against TP53 deserve particular attention. Approximately $20 \%$ of high grade ovarian cancers are associated with autoantibodies to wild type p53, but studies have not yet been performed with autoantibodies to specific mutant proteins. In studies to date, human target proteins have been expressed in bacteria or insect cells. Expression of human protein targets in human cells could provide additional authentic epitopes with appropriate conformation and glycosylation.

Sensitive imaging is critical to two stage screening strategies. Detection of small lesions in the ovary and the Fallopian tube is required. Nowosinska et al., review the use of FDG-PET/CT as a biomarker in ovarian cancer [20]. FDG-PET/CT has produced a sensitivity of $87-100 \%$ for primary diagnosis of ovarian cancer in women with an adnexal mass, but has difficulty in detecting masses $<5 \mathrm{~mm}$ in diameter or those mucinous and borderline cancers with low metabolic activity. The expense of FDG PET/CT also poses a limitation for cost-effective screening. One novel technology may provide several orders of magnitude improvement in sensitivity. Superconducting Quantum Interference Device (SQUID) sensors can detect small numbers of magnetic nanoparticles labeled with specific antibodies [21]. In admixture with normal cells in vitro and in xenografts in vivo, as few as $10^{5} \mathrm{SKOv} 3$ ovarian cancer cells can be detected using magnetic nanoparticles labeled with anti-CA125 antibodies (ER Flynn, personal communication).

Whether the behavior of hereditary ovarian cancers predicts the behavior of sporadic disease is clearly debatable. While CA125 can provide very little lead time in hereditary ovarian cancer where normal biomarker levels have been observed 3 months prior to diagnosis of widely metastatic disease, CA125 can begin to rise 2 years prior to diagnosis of sporadic disease. In the UKCTOCS trial, the prevalence of ovarian cancer was twice the expected incidence [5], once again consistent with two years of lead time for sporadic lesions. Thus, annual screening for sporadic disease may be feasible.

Both hereditary and sporadic high grade serous cancers exhibit mutations of $\mathrm{p} 53$, but it is not certain that 
they arise at comparable times in the pathogenesis of the disease. In hereditary disease, mutation of p53 and complete loss of BRCA function are very early events. Studies of prophylactic oophorectomies in carriers of BRCA mutations have documented p53 mutations in subserosal ovarian cysts that contain dysplastic, but not yet invasive epithelial cells. Loss of BRCA is observed in the smallest invasive cancers. Similarly, the fimbriae of Fallopian tubes from BRCA mutation carriers exhibit areas of p53 mutation without invasive lesions. Although the pathogenesis of sporadic disease is not as well understood, it is possible that p53 mutation and the development of BRCAness are later events.

Finally, we may have set our goals for early detection too high. Many investigators have focused only on stage I disease, based upon the assumption that once disease had metastasized, there would be little benefit in early detection. Detecting stage II and small volume stage III disease that could be completely removed surgically should, however, also translate into improved survival. In studies of cytoreductive surgery, the best prognosis has been found when no gross residual disease remained at the conclusion of the operation. A great deal depends, as well, on the sensitivity of high grade serous carcinoma to platinum and taxane based chemotherapy. Earlier detection may translate into a further improvement in 5 year survival, but may not change the plateau on the survival curve. While an increased rate of cure is clearly preferable, improved survival is still a worthy goal. The ultimate cure of ovarian cancer is likely to depend upon the simultaneous application of both earlier detection and more effective personalized therapy.

Prognostic biomarkers might contribute to personalizing therapy of ovarian cancer. A variety of molecular alterations have been associated with better or worse progression free or overall survival. In this issue, Huang et al., have carefully reviewed a substantial number of diverse biomarkers that have been correlated with outcomes for ovarian cancer patients [22]. Most prognostic studies have concerned individual biomarkers. Many have not been adequately validated with independent retrospective sample sets or with prospective trials. As the authors suggest, there is pressing a need to determine how combinations of biomarkers interact by assaying biomarker panels in the same set of clinical samples and integrating multiple independent biomarkers using mathematical models.

To date, prognostic biomarkers for ovarian cancer have had little impact on clinical practice. Most patients undergo both cytoreductive surgery and combi- nation chemotherapy, regardless of stage. A small fraction of patients with stage I low grade lesions can be managed with surgery alone. At present, this decision depends largely on tumor stage and grade. Biomarkers could help to define this subset of patients with greater accuracy. To identify patients who might benefit from chemotherapy, biomarkers associated with type I and type II cancers could be measured in archival tissue from patients who had been treated with surgery alone. One could test the prediction that patients with early stage disease which expressed type II markers would have a worse prognosis and be more likely to relapse. Prospective validation of biomarkers in this setting will, however, be difficult to achieve given the small number of patients with low grade stage I disease.

For patients with more advanced high grade ovarian cancer diagnosed in stage III and IV, the clinical application of prognostic biomarkers is also problematic. To date, most associations of biomarkers with outcome have been statistically significant, but have not been sufficiently precise to be used in the care of individual patients. If prognosis could be defined with greater accuracy using a panel of biomarkers, it is still not clear how they could be utilized for personalized care. Given currently available therapy, patients with the most favorable prognosis still do poorly. This contrasts with breast cancer where disease can often be cured and survival in a fraction of patients affected dramatically by chemotherapy. Prognostic gene profiles such as Oncotype $\mathrm{Dx}^{\mathrm{TM}}$ [23] and MammaPrint ${ }^{\mathrm{TM}}$ [24] have been used to identify breast cancer patients with a poor prognosis who would benefit from chemotherapy in addition to hormonal therapy, avoiding chemotherapy and unnecessary toxicity in patients with a good prognosis. In managing ovarian cancer, one might imagine treating patients with a poor prognosis more aggressively. In trials to date, however, adding additional active drugs such as gemcitabine, pegylated liposomal doxorubicin or topotecan to the conventional regimen of carboplatin and paclitaxel has not improved progression free or overall survival [25]. One could also imagine the use of maintenance therapy in patients with a poor prognosis, but improved overall survival has not yet been demonstrated for treatment beyond six courses of carboplatin and paclitaxel. Consequently, while prognostic biomarkers could inform the design of trials with new agents, they are not readily applicable to conventional care.

Effective predictive biomarkers will be essential for personalizing therapy. During primary therapy, $70 \%$ of ovarian cancers respond to platinum compounds, 
but only $42 \%$ respond to taxanes based on results of GOG132 [26]. Consequently, more than half of patients are exposed to the toxic effects of paclitaxel without objective benefit. Reliable biomarkers that predict resistance to paclitaxel therapy would permit the use of other agents in combination with carboplatin.

A number of targeted therapies have been evaluated in GOG172, generally producing a response rate of $<$ $10 \%$ [27]. Reliable biomarkers that predict response to these particular agents could identify the small fraction of individuals who would benefit from each available drug. Moving forward, it will be essential to biopsy recurrent ovarian cancers immediately before treatment to match molecular abnormalities with particular targeted drugs. Genetic defects that drive growth of cancer cells or permit their survival are likely to provide relevant predictive biomarkers. In the immediate future, mutational analysis is most likely to aid in management of low grade serous, mucinous and endometrioid cancers that contain actionable alterations of Ras, Raf and PTEN for which specific therapies are being evaluated in clinical trials. Recently published data from the Cancer Genome Atlas Project suggest that almost all high grade ovarian cancers have mutations of TP53 and $40-50 \%$ have defects in homologous recombination repair with 1) germ line or somatic mutations of $B R C A 1$ or $B R C A 2,2$ ) silencing of $B R C A 1$ by promoter methylation, or 3) mutation of other genes that affect the pathway [28]. While we do not yet have effective strategies to target mutant TP53, PARP inhibitors produce a high response rate in patients with germ line mutations of $B R C A 1$ or $B R C A 2$ [29]. Clinical trials are being planned to determine whether cancers that have acquired BRACAness through other mechanisms will respond to PARP inhibitors that block alternative pathways of DNA repair [4]. As the technology develops to measure DNA copy number abnormalities in clinical biopsies under CLIA approved conditions, inhibitors already exist for 22 genes that map to areas of amplification in high grade serous ovarian cancers. Clinical trials will be required to determine whether matching targeted agents to amplified genes will improve rates of response. Recent studies from M.D. Anderson suggest that matching mutations which activate signaling pathways with appropriately targeted drugs can improve response rates in phase I trials with multiple cancer types from $6-8 \%$ to $29 \%$, lengthening time to progression and overall survival [30].

One of the greatest unmet clinical needs is to define predictive biomarkers for response to bevacizum$\mathrm{ab}$ which binds VEGF and mediates anti-vascular ther- apy when administered alone or in combination with cytotoxic drugs. Among more than a dozen targeted therapies evaluated for management of ovarian cancer, bevacizumab has the highest rate of objective response $(20 \%)$ and the greatest fraction of patients with stable disease ( $40 \%$ for 6 months). In randomized trials for primary disease (GOG 218 and ICON7) and for recurrent platinum sensitive disease (OCEANS and GOG213), significant improvement of progression free survival has been observed by the addition of bevacizumab to conventional chemotherapy [31]. Overall survival has not, however, been affected and bevacizumab has had to be administered for 9-15 months after completion of cytotoxic chemotherapy to achieve a 4 month prolongation of progression free survival. Given the cost of bevacizumab and the fact that more than half of patients do not benefit from treatment, biomarkers that accurately predict resistance to the drug would find immediate clinical use. In this issue of Cancer Biomarkers, Li, Sassaroli and colleagues provide a comprehensive catalog of the molecules that are differentially expressed in ovarian cancer vasculature [32]. Among these proteins, new therapeutic targets will almost certainly be found, but proteins expressed in approximately half of ovarian cancers might also provide predictive biomarkers for anti-vascular therapies, including bevacizumab.

Given new technologies to identify biomarkers and an increasing knowledge of diverse mechanisms that drive and sustain different ovarian cancers, the next decade should witness significant advances in early detection and personalized therapy. Steady support of relevant translational research and the prompt development of clinical trials which apply molecular diagnostics to screening and to targeted therapy will determine how rapidly we progress.

\section{Acknowledgements}

This work was supported by funds from the M.D. Anderson SPORE in Ovarian Cancer NCI P50 CA83639, the M.D. Anderson CCSG NCI P30 CA16672, The National Foundation for Cancer research, The Ovarian Cancer Research Fund and philanthropic support from Golfers Against Cancer, the Tracey Jo Wilson Foundation, the Mossy Foundation, the Zarrow Foundation and Stuart and Gaye Lynn Zarrow. 


\section{Conflict of interest}

Robert C. Bast receives royalties for the discovery of CA125 and serves on the advisory board for Fujurebio Diagnostics, Vermillion, Inc and Illumina, Inc.

\section{References}

[1] A. Jemal, R. Siegel, E. Ward, Y. Hao, J. Xu and M.J. Thun, Cancer Statistics 2009, CA Cancer J Clin 59 (2009), 225-249.

[2] R.C. Bast, Jr., B. Hennessy and G.B. Mills, The Biology of Ovarian Cancer: New Opportunities for Translation, Nature Rev Cancer 9 (2009), 415-428. PMID: 19461667.

[3] The Cancer Genome Atlas Research Network, Integrated genomic analyses of ovarian carcinoma, Nature 474 (2011), 609615.

[4] R.C. Bast and G.B. Mills, Personalizing therapy for ovarian cancer: BRCAness and beyond, JClin Oncol 28 (2010), 35453548. PMID: 20547987.

[5] U. Menon, A. Gentry-Maharaj, R. Hallett, A. Ryan, M. Burnell, A. Sharma et al., Sensitivity and specificity of multimodal and ultrasound screening for ovarian cancer, and stage distribution of detected cancers: results of the prevalence screen of the UK Collaborative Trial of Ovarian Cancer Screening (UKCTOCS), Lancet Oncol 10(4) (2009), 327-340.

[6] K.H. Lu, S. Skates, T. Bevers, O. Adeyinka, W. Newland, R. Moore, L. Leeds, L. Harris, S. Harris, H. Fritsche, R.C. Bast Jr., A prospective U.S. ovarian cancer screening study using the risk of ovarian cancer algorithm (ROCA), Proc Amer Soc Clin Oncol 28 (2010), (A\#5003).

[7] D. Rosen, L. Wang, J.N. Atkinson, Y. Yu, K. Lu, E.P. Diamandis, I. Hellstrom, S.C. Mok, J. Liu, R.C. Bast, Jr., Potential markers that complement expression of CA 125 in epithelial ovarian cancer, Gynecol Oncol 99 (2005), 267-277. PMID: 16061277.

[8] K.D. Rodland and N.J. Maihle, Searching for a system: The quest for ovarian cancer biomarkers, Cancer Biomark 8 (2011), 223-230.

[9] A.B. Ramirez and P.D. Lampe, Discovery and validation of ovarian cancer biomarkers using high density antibody microarrays, Cancer Biomark 8 (2011), 293-307.

[10] K.L. Abbott, Glycomic analysis of ovarian cancer: Past, present and future, Cancer Biomark 8 (2011), 273-280.

[11] C. Balch and K.P. Nephew, The role of chromatin, microRNAs and stem cells in ovarian cancer, Cancer Biomark 8 (2011), 203-221.

[12] C.D. Roberson, S. Atay, C. Gercel-Taylor and D.D. Taylor, Tumor-derived exozomes as mediators of disease and potential biomarkers, Cancer Biomark 8 (2011), 281-291.

[13] B.N. Nolen and A.E. Lokshin, Screening for ovarian cancer: Old tools, new lessons, Cancer Biomark 8 (2011), 177-186.

[14] P.O. Brown and C. Palmer, The preclinical natural history of serous ovarian cancer: defining the target for early detection, PLoS Med 6 (2009), e1000114.

[15] D. Badgwell and R.C. Bast, Jr., Urinary Mesothelin Provides Greater Sensitivity for Early Stage Ovarian Cancer than Serum Mesothelin and Urinary hCG Free Beta and Beta Core Fragment, Gynecol Oncol 106 (2007), 490-497. PMID: 17532030.

[16] N. Anderson, Y. Bermudez, D. Badgwell, B.O. Saunders, R.
Chen, G.D. Wilbanks, S.V. Nicosia, R.C. Bast, Jr. and P.A. Kruk, Urinary levels of Bcl-2 are elevated in ovarian cancer patients, Gynecol Oncol 112 (2009), 60-67. PMID: 19007973.

[17] B. Nolen, A. Lomakin, R. Bast, Jr., K. Lu, W.E. Grizzle, N.C. Jhala, M.A. Eloubeidi, J.D. Christein, J.P. Arnoletti, D.K. Oleschlager, S.M. Vickers, A. Marrangoni and A. Lokshin, Urine biomarkers outperform serum biomarkers in the diagnosis of different cancers, Proc Amer Assoc Cancer Res $\mathbf{5 1}$ (2010), (A\#4567).

[18] K.L. Terry, P.M. Sluss, S.J. Kates et al., Blood and urine markers for ovarian cancer: A comprehensive review, Dis Markers 20 (2004), 53-70.

[19] M. Chatterjee and M.A. Tainsky, Autoantibodies as biomarkers for ovarian cancer, Cancer Biomark 8 (2011), 187-201.

[20] E. Nowosinska, S. Avril, I. Murray, T. Szyszko and N. Avril, FDG-PET/CT as a molecular biomarker in ovarian cancer, Cancer Biomark 8 (2011), 167-175.

[21] N.L. Adolphi, D.L. Huber, H.C. Bryant, T.C. Monson, D.L. Fegan, J. Lim, J.E. Trujillo, T.E. Tessier, D.M. Lovato, K.S. Butler, P.P. Provencio, H.J. Hathaway, S.A. Majetich, R.S. Larson and E.R. Flynn, Characterization of single core magnetic nanoparticles for magnetic imaging by SQUID relaxometry, Phys Med Biol 55(19) (7 Oct 2010), 5985-6003. Epub 2010 Sep 21.

[22] J. Huang, W. Hu and A.K. Sood, Prognostic biomarkers in ovarian cancer, Cancer Biomark 8 (2011), 231-251.

[23] S. Paik, S. Shak and G. Tang, A multi-gene assay to predict recurrence of tamoxifen-treated node negative breast cancer, New England J Med 351 (2004), 2817-2826.

[24] M.E. Straver, A.M. Glas, J. Hannemann et al., The 70-gene signature as a response predictor for neoadjuvant chemotherapy for breast cancer, Breast Cancer Res Treat 119 (2010), $551-558$.

[25] M. Bookman, M.F. Brady, W.P. McGuire, P.G. Harper, D.S. Alberts, M. Friedlander, N. Colombo, J.M. Fowler, P.A. Argenta, K. De Geest, D. Mutch, R.A. Burger, A.M. Swart, E.L. Trimble, C. Accario-Winslow and L.M. Roth, Evaluation of new platinum-based treatment regimens in advanced-stage ovarian cancer: A phase III trial of the Gynecologic Cancer Intergroup, J Clin Oncol 27 (2009), 1419-1425.

[26] F.M. Muggia, P.S. Braly, M.F. Brady et al., Phase III randomized study of cisplatin versus paclitaxel versus cisplatin and paclitaxel in patients with suboptimal stage III or IV ovarian cancer: a gynecologic oncology group study, J Clin Oncol 18 (2000), 106-115.

[27] J.A. Ledermann and F.A. Raja, Targeted trials in ovarian cancer, Gynecol Oncol 119 (2010), 151-156.

[28] The Cancer Genome Atlas Research Network. Integrated genomic analyses of ovarian carcinoma, Nature 474 (2011), 609615.

[29] P.C. Fong, D.S. Boss, T.A. Yap et al., Inhibition of Poly (ADP Ribose) Polymerase in tumors from BRCA mutation carriers, New England J Med 361 (2009), 123-134.

[30] A.M. Tsinmberidou et al., Personalized medicine in a phase I trials program: The M.D. Anderson initiative, J Clin Oncol 29(18S) (2011), 787s (Abstract \#CRA2500).

[31] R.N. Eskander and L.M. Randall, Bevacizumab in the treatment of ovarian cancer, Biologics 5 (2011), 1-5.

[32] C. Li, D. Sassaroli, X. Chen, J. Hu, R. Sandaltzopoulos, Y. Omidi and G. Coukos, Tumor Vascular Biomarkers: New Opportunities for Cancer Diagnostics, Cancer Biomark 8 (2011), 253-271. 